\title{
MAGNETIC FuSION ENERGY RESEARCH
}

\section{A SUMMARY OF ACCOMPLISHMENTS}

U.S. Department of Energy

Office of Energy Research - Office of Program Analysis

Washington, DC 20585

\section{December 1986}

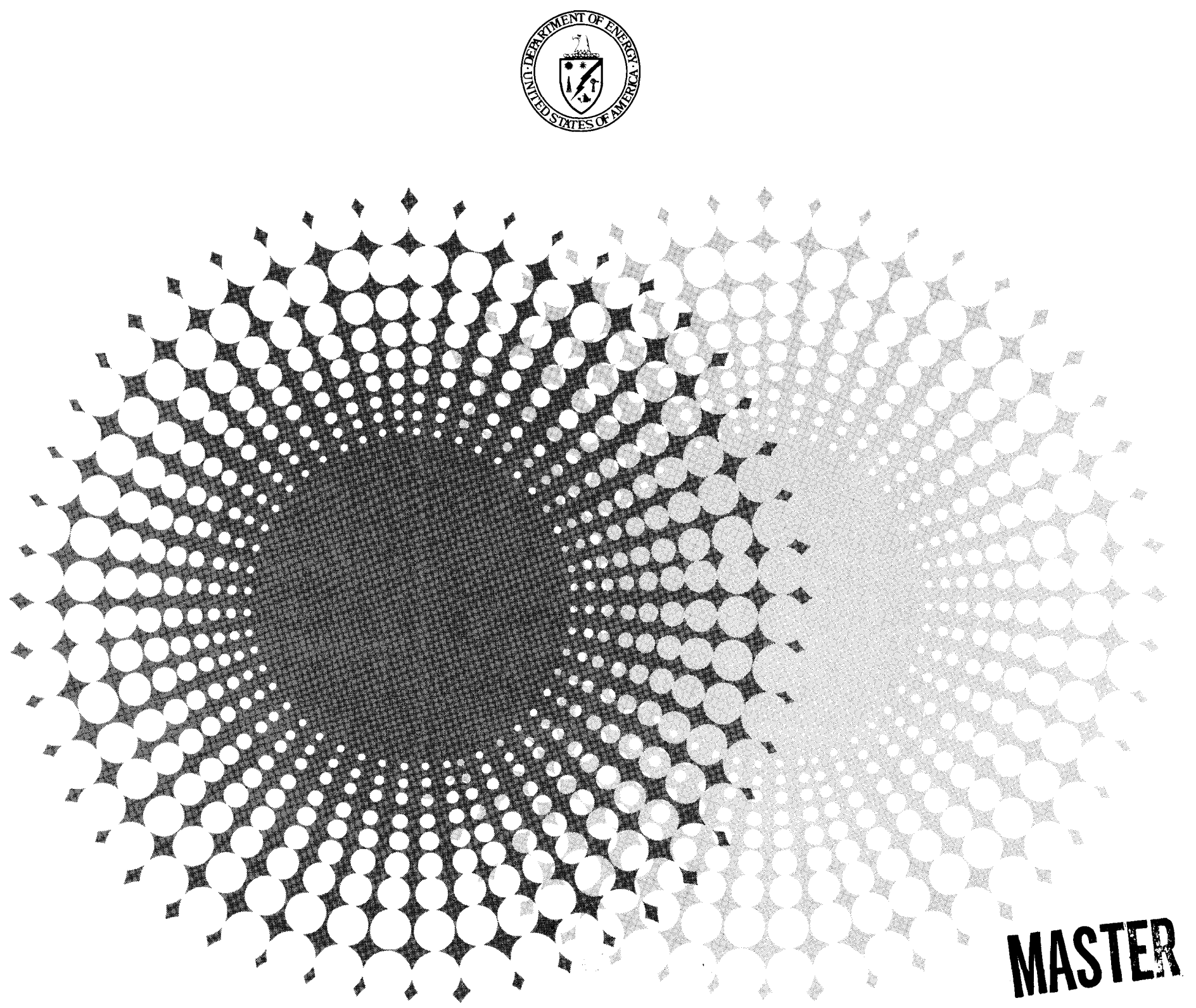

DOE/ER- -0297

DE87 004222 


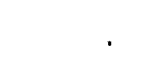




\section{DISCLAIMER}

This report was prepared as an account of work sponsored by an agency of the United States Government. Neither the United States Government nor any agency thereof, nor any of their employees, make any warranty, express or implied, or assumes any legal liability or responsibility for the accuracy, completeness, or usefulness of any information, apparatus, product, or process disclosed, or represents that its use would not infringe privately owned rights. Reference herein to any specific commercial product, process, or service by trade name, trademark, manufacturer, or otherwise does not necessarily constitute or imply its endorsement, recommendation, or favoring by the United States Government or any agency thereof. The views and opinions of authors expressed herein do not necessarily state or reflect those of the United States Government or any agency thereof. 



\section{DISCLAIMER}

Portions of this document may be illegible electronic image products. Images are produced from the best available original document. 

Fusion energy is the internal power source of the sun and the stars. Resisting natural forces of separation, lighter elements, such as hydrogen and helium, are forcibly joined together to form the nucleus of a heavier element of slightly less mass than the sum of its original parts. In accord with Einstein's now familiar equation, this small difference in mass is converted into an enormous amount of energy, which we ultimately observe as heat and light from the sun.

In the stars, this process is made possible by the extraordinary physical conditions under which matter in the core of a star is compressed to high densities and heated to high temperatures by the nearly unimaginable gravitational weight of the rest of the star. Today, scientists on earth hope to find equivalent conditions with an array of large machines.

Although a practical fusion energy device is still well in the future, the potential benefits are enormous. The fuel could be extracted from ordinary sea water in virtually inexhaustible supply. The heat and power produced could provide energy for future generations long after the earth's inventory of conventional fossil fuels had been depleted.

In this relatively brief document, some of the more important contributions of the research program needed to establish the scientific and technical base for fusion power production are discussed. The number of contributions has grown steadily and at an increasing pace. I have every confidence that this research program will meet its future challenges.

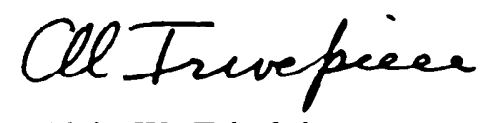

Alvin W. Trivelpiece

Director, Office of Energy Research 



\section{Contents}

1

\section{3}

3

4

5

5

\section{Introduction}

Origins of the Research Program

Evolution of the MFE Program

The Present and Beyond

\section{Fusion Science \\ Improvement of Plasma Behavior \\ Development of Theoretical Understanding \\ Construction of Experimental Devices}

\section{Fusion Technology}

Confinement Technologies

Auxiliary Plasma Heating Technologies

Plasma Fueling Technologies

Materials Technologies

Reactor Concepts

International Dimensions

Superconducting Magnet Development

Plasma/Wall Interactions

Doublet-III

International Tokamak Reactor

Materials Research 
Today, in major laboratories of the United States, the European Economic Community, Japan, and the Soviet Union, a new energy technology is being developed for world use. The technology is that of magnetic fusion energy, where the word magnetic refers to the technique of holding the fuel in place by means of strong magnetic fields. The starting fuel will be forms of the element hydrogen.

The final form or forms of this new energy source are not yet fixed. They will be determined over the next few decades, first by scientific results and Governmental research policy, and later by research and investment decisions in the private sector. The availability of magnetic fusion for use by the Nation's energy producers will depend strongly upon the technology options available and when they become available.

The purpose of this brief narrative is to foster an awareness of the publicly funded Magnetic Fusion Energy Research Program and of its progress towards establishing the base of scientific and technical information required for commercial development of fusion energy. This program, administered by the Office of Fusion Energy, is one of many research activities of the Department of Energy (DOE). The program has now completed three decades of accomplishment.

Over the years, this program has been sponsored by DOE and its predecessors which are responsible for the National energy mission. Its evolution has been a reflection of changes in time, in public priorities, and in legal mandate. But, throughout this evolvement, there has remained a consistency of purpose: to seek a fundamental understanding of the fusion energy processes observed in the sun and stars; to establish the body of theoretical, experimental, and engineering knowledge necessary to use these processes to generate energy.

The story of this research program is told through its accomplishments. Several of the more significant accomplishments have been chosen here for illustrative purposes. Following a brief review of the program's origins, evolution, and current mission, the accomplishments are grouped and reviewed in the two broad categories of fusion science and fusion technology. This is followed by a review of international cooperative research activities which are conducted to further advance the program's effectiveness. Explanatory material is woven into the story for the benefit of most readers, who will be unfamiliar with the science and technology of fusion. 



\section{Origins of the Research Program}

The Department of Energy's (DOE's) Magnetic Fusion Energy (MFE) Program is aimed at capturing the fusion processes that power the sun and the stars and harnessing them on earth. The program is building the scientific and technical base that must be in place for commercial development of magnetic fusion energy as a major new source of power.

The long-term attractiveness of fusion as an energy option is based on two key factors: the potential of a readily available, vast and secure fuel reserve in sea water, and the intrinsic environmental and safety advantages of fusion energy. Fusion is one of the very few energy options that could provide mankind's energy into the distant future and meet the Nation's high National standards for public health and safety.

The MFE Program, now managed by DOE's Office of Fusion Energy, had its origins in the early 1950's. It began with grassroots enthusiasm for fusion research, initiated by scientists connected with the National Laboratories. It soon became evident, however, that achieving the capability to produce fusion power on earth would take decades of research and development. Despite the extraordinary economic potential of developing an essentially inexhaustible supply of energy, launching such an extended research and development effort required investment by the Federal Government. In July 1951, the Atomic Energy Commission awarded a grant to Princeton University to conduct studies on magnetic fusion; and with that, the
United States program in magnetic fusion was off the ground.

\section{Evolution of the MFE Program}

The period from 1951 to 1958 saw tremendous scientific creativity and growth in the development of magnetic fusion. Emphasis was on laboratory experimentation directed toward constructing a prototype fusion device. Between 1952 and 1955, the performance of experimental devices improved a thousandfold but fell short of that required for practical fusion power. In the late 1950's, United States, British, and Soviet magnetic fusion programs were declassified under the United States Atoms for Peace Initiative. This fostered an environment for international cooperation and open scientific exchange that has characterized and nourished the program ever since.

By the end of 1957, British researchers, with whom United States scientists were cooperating and sharing results, reported experimental results suggesting that one of their fusion machines had reached the point at which it could be scaled up to a commercial fusion power device. Unfortunately, further tests proved otherwise; and this led to a widespread realization within the fusion community that more emphasis was needed on expanding the theoretical and scientific foundations upon which fusion science was based.

Then followed a decade of scientific progress, based primarily on 
expanded research into the theories describing the behavior of the fusion plasmas which fueled the experimental devices. Fusion fuels are in the form of an ultra-hot gas called a plasma. A plasma differs from an ordinary gas in that magnetic fields can be used to control its movement and position. Researchers focused chiefly on the phenomena of plasma instabilities that had limited the success of early experiments.

Armed with an improved theory base, United States scientists reemphasized efforts to improve experimental devices. Nearly at the same time, the Soviets achieved a major performance improvement in their doughnut shaped "tokamak" device. Russian fusion researchers reported obtaining three times the performance levels that had been achieved on magnetic fusion devices in the United States. These results were verified by British scientists, who were invited to conduct experiments on the Soviet tokamak.

By 1970 the United States adopted the tokamak as its mainline device, and the program mission was significantly broadened. It provided for an orderly series of steps to a final, commercially usable product with a demonstration plant planned for 1995. More attention was given to the development of the components required to design and build a reactor. In support, plasma investigations were focused on the needs of the larger machines that formed the program's backbone: the tokamak on the one hand, and one or two practical and vigorous competitors on the other.

In 1977 research and development of MFE was assigned to DOE. At that time, the leaders of the new Department revised the Nation's energy research and development program, and with it, the MFE Program schedule.

Federal policy changes in the early 1980 's produced additional modification in the MFE Program and redefined the program goals. The new National Energy Policy Plan in 1981 stated that the goal of longterm research and development in energy is to carry promising technological innovations to the point where private enterprise can reasonably assess their potential. In keeping with the new directions in policy, plans for Government participation in the commercialization of MFE and Federal support of a demonstration project have been deemphasized. The goal of the program now focuses more narrowly on providing the necessary scientific and technological base for fusion energy.

\section{The Present and Beyond}

Today the rescarch and development effort in magnetic fusion seeks to gain a fundamental understanding of the fusion energy processes observed in the sun and stars; to establish the body of theoretical, experimental, and engineering knowledge necessary to apply these processes to generating power on earth; and to do so in a way that will be useful to the Nation's energy producing industries and meet our high National standards for public health and safety.

The Office of Fusion Energy supports advanced research facilities and talented scientific personnel from universities, National Laboratories, and industry that are among the strongest in the world. Participation of the full spectrum of available scientific talent provides innovative ideas, major technical advances, and manpower training to further United States technological growth. At the approximately 26 universities involved in magnetic fusion research, a total of 1,100 Ph.D.s have been trained in association with fusion research. Many talented scientists and engineers with roots in the magnetic fusion program are making outstanding contributions in other fields, including defense and commercial applications, space and astrophysical plasma physics, computational physics, and materials science. The participation of industry accelerates the transfer of fusion technology to the private sector and attracts industrial investment to fusion related activities.

The MFE Research Program has made remarkable strides toward bringing the Nation closer to a secure and virtually limitless source of energy. Although impressive achievements have been made, the greater goal of fostering commercial development remains to be attained.

Early in the program, the basic laboratory objective appeared deceptively simple: heat a pressurized container of plasma fuel to the point of fusion, and then contain and sustain the reaction. Only after a decade of intense scientific creativity did fusion researchers realize that bringing about a controlled fusion reaction was one of the most complex and challenging feats ever attempted and that it might lie well beyond the theory and experimental capability they could foresee in the 1950's.

Today that situation has changed. The scope and complexity of the problem and the requirements for solving it are much better understood. Fusion technology has advanced immensely; and, based on the foundations of scientific and technical accomplishments described in this document, experiments are well underway to answer the key question: how can we best harness fusion power on earth? 


\section{Fusion Science}

\section{Objective:}

To be able to predict, control, and optimize the behavior of plasma for a fusion power reactor.
For more than 30 years, the improvement in experimental capabilities and physical understanding of fusion plasmas has been remarkably steady. The results are now evident. In each crucial area of plasma behavior, scientists have achieved the performance levels they need in order to attain the program objective. What remains, however, is to achieve them all in a single experiment. The advances of the last decade have brought the research program to a point where such an experiment is now being undertaken, based on the solid foundations of plasma science developed in the research program.

The scientific achievements of the program are shown by improved plasma behavior, development of theoretical understanding, and construction of experimental devices.

\section{Improvement of Plasma Behavior}

The MFE Program seeks to produce a fusion reaction which produces more energy than is required to bring it about. The program has already succeeded in producing a limited amount of energy in experimental fusion devices, but to establish the ultimate scientific feasibility of fusion power, it is necessary to get at least as much energy out as is put in. Improvements in plasma behavior are measured chiefly in terms of the relationship between energy in and energy out and in how efficiently the magnetic field can confine the plasma fuel while fusion occurs (Beta).
Energy Gain - In the fusion community, the relationship between energy out and energy in is called energy gain. It is expressed as the ratio of the output energy produced by the plasma to the input energy needed to produce and maintain the plasma. An energy gain of 1.0 describes break-even, the condition under which equal amounts of energy are put into the system as are produced by the system. The higher the energy gain the more attractive the energy source. Proximity to an energy gain equal of 1.0 (breakeven) serves as a benchmark of progress towards the experimental goal of scientific feasibility.

In 1985 the Tokamak Fusion Test Reactor (TFTR) achieved an energy gain equivalent to 0.12 , or approximately one-eighth of break-even. (Since experimental fusion devices are powered by deuterium fuel rather than a deuterium/tritium mixture, an energy gain attained experimentally is somewhat less than an "equivalent" energy gain that would have resulted had radioactive tritium been added to the fuel mixture.) This represents a six-fold increase over the peak energy gain attained on TFTR's predecessor, the Princeton Large Torus (PLT).

The main plasma parameters which fusion researchers manipulate as they strive to increase energy gain are temperature, density, and confinement time. Since energy gain increases rapidly with increases in these plasma parameters, they commonly serve as interim measures of the experimental progress toward achieving a higher energy gain. 
Plasma Confinement (N-tau) - The fuel confinement time (seconds) and density (particles/cubic centimeter) of ionized nuclei in the plasma combine in a factor termed $\mathbf{N}$-tau. A British scientist, J.D. Lawson, determined that for break-even to occur, $\mathrm{N}$-tau has to be 100 trillion and the plasma temperature must be 70 million degrees Celsius. There is a permissible trade-off, however, between plasma density and confinement time-the greater the density, the less the confinement time and vice versa. The most difficult challenge of the past 30 years has been to reach an N-tau of 100 trillion. In 1983 United States fusion scientists reached it on the Alcator $\mathrm{C}$ machine and in 1985 duplicated their success on the TFTR.

Plasma Temperature - The temperatures needed for break-even as well as the higher temperatures needed for economic reactor operation have both been achieved. For break-even to occur, a plasma has to reach at least 70 million degrees Celsius; and for a power reactor, which will require an energy gain greater than 1.0, 100 million degrees Celsius is needed. Fusion research passed the first goal in 1979 when the PLT produced headlines around the world and a temperature of 75 million degrees Celsius. In 1985 the 100 million degree Celsius threshold was reached on the TFTR, and in 1986 that record high was extended beyond the 200 million degrees Celsius mark.

Magnetic Confinement Efficiency (Plasma Beta) - An indicator termed beta is used by the fusion community to provide another important estimate of progress towards a commercial fusion power plant. Beta addresses the relationship between the magnetic forces confining the plasma and the forces driving it to expand as it is heated up. Beta is, then, a measure of the efficiency (expressed as a percentage) with which the magnetic field confines the plasma fuel while fusion occurs.

All investigations into the form of a possible fusion power reactor focus on beta. Since one-fourth to one-third of the cost of a commercial reactor may be attributable to the magnet systems, beta values are directly related to the economics of fusion power production. For this reason, beta will play an important role in future assessments of the engineering and economic feasibility of fusion reactors. High plasma beta values imply high power levels for each dollar of investment and lead to better power plant economics.

Several tokamak experiments have produced data on beta. Values of 5 percent have been achieved, approaching the 7 to 10 percent value estimated as sufficient for an operating reactor.

\section{Development of Theoretical Understanding}

The outstanding recent improvements in optimizing plasma behavior have been made possible by rapid advances in plasma physics, and computational physics. Plasma confinement now rests on a solid theoretical understanding of the principal phenomena affecting the dynamics of plasmas. To an ever increasing extent, advances in confinement result from some new insight into the theoretical properties of a particular confinement configuration. And, there is a clear definition of the major remaining theoretical unknown-energy losses due to localized disturbances within the plasma-although the scientific tools to understand these phenomena are not yet in hand.

Plasma Physics - More than 99 percent of the matter in the universethe sun, the stars and galaxies, plus the material found in interstellar space-is in the form of plasmas similar to those found in fusion devices. One of the most significant contributions to the basic sciences made by fusion research relates to the development of the scientific discipline of plasma physics.
When the MFE Program began in the 1950's, plasma physics-the study of the multiple and complex interactions of charged particles with one another and with their own and other electric and magnetic fieldswas in its infancy and being developed primarily by astrophysicists. When fusion researchers encountered inexplicable phenomena associated with magnetically confined plasma fuel in their early experiments, they realized that the development of a fusion reactor would require extension of the infant science of plasma physics. Plasma physics encompasses such fusion relevant topics as plasma equilibrium and stability, confinement, instabilities, turbulence, chaos, and the propagation of particles and radio waves through the plasma.

The broad-based fundamental studies of magnetically confined plasmas over the past two decades have solidified the underpinnings of the relatively new science of plasma physics. Nurtured by the contributions of fusion researchers, plasma physics has since grown to maturity and become the theoretical foundation upon which not only contemporary fusion theory rests, but also modern astrophysics and space physics as well. While plasma physics did not originate in the fusion program, the development of plasma physics into a mature and productive science is a direct product of the fusion effort.

Equilibrium and Stability Theory Theories prevailing in the 1950's could neither predict nor adequately explain the unexpected phenomena resulting from early attempts to confine plasma fuel within a magnetic field and heat it to fusion conditions. The primary uncertainties revolved around efforts to confine the plasma to the initial shape desired and then to contain its heat as the plasma nears fusion conditions.

Pioneering advances in plasma theory by fusion researchers have explained the early laboratory phenomena and provided much of the basic understanding needed to optimize 
plasma behavior. Plasma theory explains what happens when a plasma moves within electric and magnetic fields. Two fundamental constituents of plasma theory owing primarily to the MFE Program for their development are equilibrium theory and stability theory.

Equilibrium Theory - Fusion equilibrium is the term used to refer to the geometrical configuration consisting of the fusion plasma core, the surrounding magnetic field and vacuum chamber as well as the external magnetic field coils which produce the confining fields. Equilibrium theory explains how the fusion plasma behaves in the presence of the other elements comprising the fusion equilibrium.

Today, a fusion equilibrium is designed through the use of computer programs based on equilibrium theory rather than by experimental trial-and-error as in the early fusion investigations. These programs describe with great precision how to obtain the exact plasma shape needed for a given experiment. They specify the size and shape of the magnets, how to arrange them in relation to other components comprising the plasma equilibrium, and at what electrical currents to operate them.

Experimental results over the past 15 years have validated equilibrium theory and the programs based on it. Foremost among the benefits of equilibrium theory is that expensive experimental reactor magnets (and future power plant magnets) can be fabricated with the certainty that they will produce the plasma shapes and positions specified. Equally important, applications of equilibrium theory enable researchers to modify plasma conditions through external adjustments so they can easily investigate different plasma shapes and locations in a fusion equilibrium without tearing it down to rearrange hardware components.

Stability Theory - Due to its physical nature, a body of plasma inherently resists confinement. Under magnetic confinement in a fusion device, this resistance shows itself in a number of undesirable ways, referred to as plasma instabilities. Plasma instabilities tend to hold down the temperature in the fusion device and allow the plasma fuel to escape confinement. Stability theory describes what will happen if a plasma at rest in the fusion equilibrium is disturbed. Applications of the theory enable researchers to better predict and control plasma behavior in the fusion equilibrium.

For the past 10 years, a particular instability, the "ballooning mode," has held down plasma beta values. A ballooning mode is similar to an aneurysm or bulge in the plasma. If it bursts, the plasma loses fuel and energy.

A recent application of stability theory predicted that susceptibility to ballooning mode instabilities in tokamaks could be controlled by altering the cross-sectional profile of the plasma. Computations based on the theory predicted that devices with elongated " $D$ "'-shaped, bean-shaped, or peanut-shaped plasma crosssections rather than the conventional circular cross-section would reduce susceptibility to the ballooning mode.

Experimental tokamaks designed to avoid ballooning have already attained beta levels which, according to fusion engineers, approach those required for commercial fusion plants. For example, the fusion program's Beta Experiment, which achieved the record breaking beta values, was designed using predictions based on stability theory, and the use of such predictions is now standard in all experiments.

Computational Physics - Fusion researchers have significantly accelerated the maturation of plasma science by using computers and mathematics with increasing sophistication. Computers of all sizes assist in theoretical calculations of increasing complexity and precision, and in the design, direction and analysis of experiments.

The computer has been a tool in the evaluation of the physical theories and of the engineering models of magnetic fusion. More recently it has become a means of developing the theories themselves.

Theoretical and computational tools for understanding plasma confinement and heating in magnetic systems have developed to the level where in many instances they can now closely model plasma behavior. From rudimentary one-dimensional models run on the earliest computers, plasma computations have

\section{Magnetic Tearing and Reconnection}

Magnetic tearing and reconnection is the phenomenon by which complex magnetic field lines spontaneously rearrange themselves into new patterns. This is a broadly observed phenomenon, which manifests itself as the tearing mode instability in fusion devices. It is a major topic of investigation by scientists because of its importance in space and astrophysics. For example, its occurrence in the earth's magnetic field triggers the spectacular Northern Lights and magnetic substorms that interfere with radio communications.

One of the major advances in general plasma physics over the last 10 years is the considerable progress that has been made in understanding magnetic line tearing and reconnection. Much of this progress results from the extensive study of the tearing mode instability in tokamaks. Recently adding to the understanding of magnetic tearing and reconnection are a number of very precise and experimentally validated computer programs developed by MFE scientists to model these effects. 
evolved to sophisticated simulations that run on today's state-of-the-art supercomputers.

Some of this progress can be explained by improvements in computer hardware, but most is attributable to the development of efficient mathematical techniques and complex computer programs, or "codes," needed to solve the physics problems. Researchers use these computations to explore fusion reactor design concepts as well as to understand basic plasma phenomena on a microscopic level.

Particle Codes - Particle codes are computer programs developed almost entirely within the fusion program that describe the path followed by individual plasma particles as they move within their own and surrounding electric and magnetic fields. Particle codes combine Newton's equations of mechanical motion, and Maxwell's equations of electromagnetic field behavior and have proved valuable in understanding a number of effects observed in plasmas that are not described well by other approaches.

The physics principles described in the particle codes are also encountered in many other scientific and engineering disciplines. In solid state physics, particle codes are used to describe the behavior of electrons in semiconductor chips, such as microcircuits. In studies of single galaxies and the phenomena of galactic clustering, astrophysicists use particle codes to better understand cosmic evolution.

National Magnetic Fusion Energy Computer Center (NMFECC) - Underlying the advancements in computational physics is the emergence of the NMFECC as one of the Nation's foremost supercomputer networks and a model that is highly regarded throughout the scientific community. Established in 1975, the NMFECC is now acknowledged as a major innovator in the management and operation of the largest and most powerful supercomputers in the world. Its data communications network, providing users convenient, 24-hour access through high speed land and satellite links, is being emulated by other scientific networks under design and construction. The Center has made a particularly significant contribution to increasing the efficient use of the supercomputer itself through development of the Cray Time Sharing System.

\section{Construction of Experimental Devices}

A particular strength of the United States fusion program has been its broad base, which includes research on several confinement systems to ensure that the maximum potential of fusion is realized. A number of different types of devices have been built, each representing a concept for assembling the energy producing core of a commercial reactor.

There are only two basic magnetic structures which have been shown to confine plasmas of fusion interest: the magnetic mirror and the magnetic torus. Each of these magnetic confinement systems has several variations. These confinement systems differ in practice by emphasizing particular principles of fusion science to improve plasma confinement or to simplify the technical requirements for producing the magnetic fields used to confine the plasma.

Tokamaks - Tokamaks are toroidal (donut shaped) devices designed to produce power in pulses (see Figure 1). Both external magnet coils and a current through the plasma provide the magnetic fields that confine the fusion fuel. All current heating methods have been tested on tokamaks.

Historically, the tokamak embodied a set of principles which were comparatively easy to implement in the laboratory. As a result, most of the scientific progress in magnetic plasma confinement has been made with tokamak confinement systems.
Today tokamaks are unique in the ability to produce for scientific study, fusion plasmas resembling those expected in a fusion power reactor. Systems based on the tokamak concept are being pursued by all International Energy Agency countries with MFE Programs and the Soviet Union.

Scientific progress on tokamak systems in the United States has been especially dramatic. The most recent tokamak device yet built by the United States, TFTR, began operating in December 1982.

Tandem Mirrors - The tandem mirror is a device in which plasma fuel is confined in a cylindrical shape. The ends of the plasma cylinder are confined by magnetic and electrostatic barriers, hence the term "magnetic mirrors." It operates in a steady state using external coils to produce magnetic fields, which confine the plasma. All present methods for heating the plasma have been tested on tandem mirrors.

In 1982 the Tandem Mirror Experiment confirmed the basic principles of the tandem mirror. The Mirror Fusion Test Facility, recently dedicated at the Lawrence Livermore $\mathrm{Na}$ tional Laboratory, is the largest tandem mirror fusion device in the world. Both the United States and Japan continue to explore tandem mirror fusion systems.

Alternate Concepts - In addition to tokamaks and tandem mirrors, several concepts for alternative confinement systems have been devised and tested in the research program, although none have been taken to the levels of development of tokamak and mirror devices. The primary objective of pursuing research on alternate concepts is to advance the study of the scientific principles of magnetic confinement which may lead to more attractive fusion devices.

The reverse-field-pinch and the stellerator/torsatron, two particularly attractive magnetic confinement 


\section{Tokamak Fusion Test Reactor}

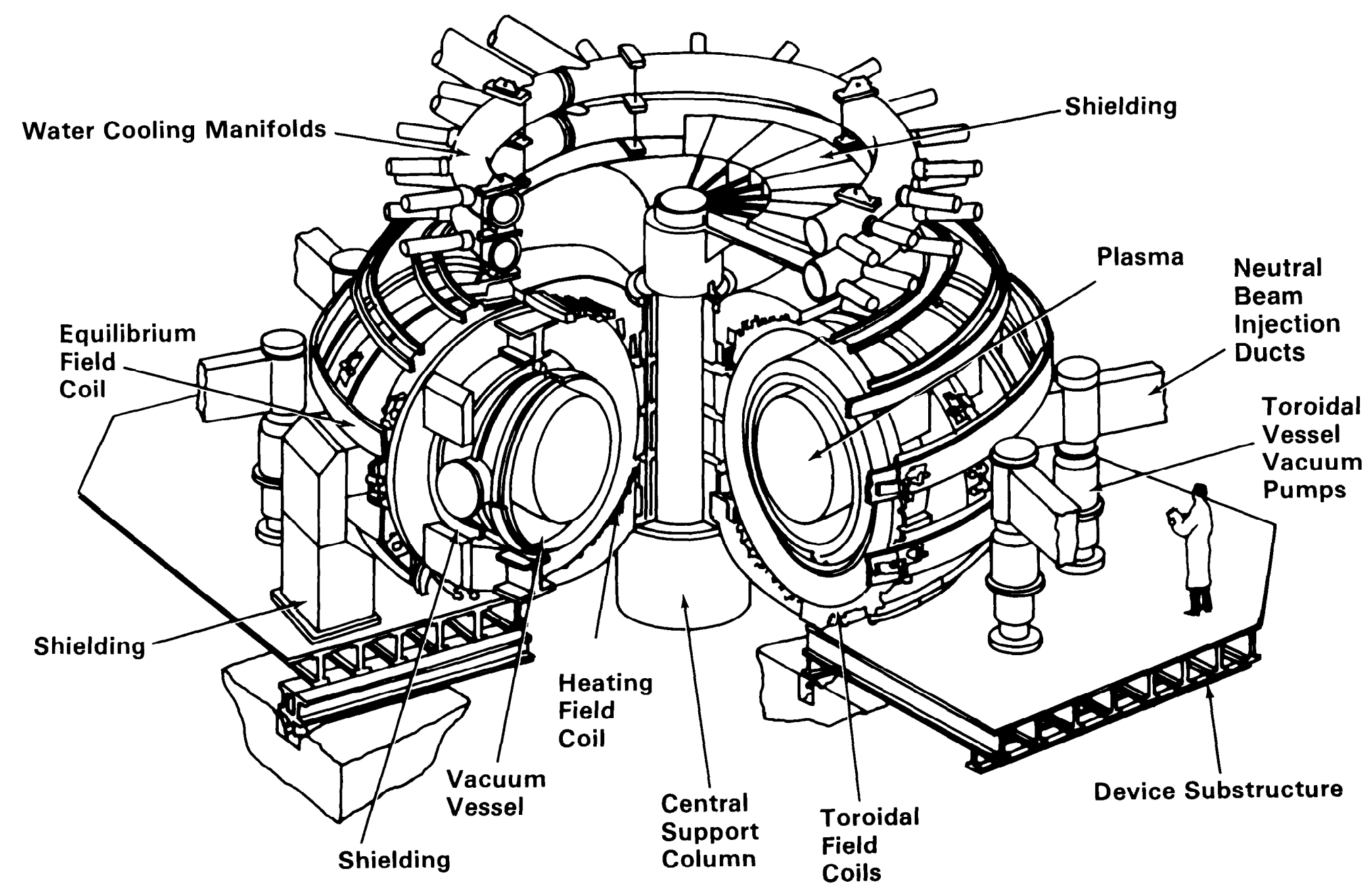

Figure 1 
configurations, have emerged successfully from initial tests and experimentation based on a number of alternate concepts. Both variations offer very efficient use of the magnetic fields and the potential for smaller, less expensive magnet systems and simpler engineering, in general, leading to less costly commercial fusion devices.

The reverse-field-pinch concept is similar in appearance to the tokamak, but the magnetic fields are applied in such a way that plasma fuel can be confined with greater magnetic efficiency. Fuel in a device based on this concept might be heated to fusion temperatures without the use of auxiliary heat sources.

The stellerator/torsatrons are toroidal devices in which the magnetic coils are wound at an angle around the toroidal plasma vessel. The advantage of this arrangement is that plasma control is simplified, and the devices can operate in steady state instead of in pulses. 


\section{Fusion}

\section{Technology}

\section{Objective:}

Show that it is possible to create the unique components and subsystems required to perform required experiments and to develop a fusion power reactor.
For magnetic fusion to become a practical energy source, scientific insights must be developed through technology and engineering into laboratory achievements and then into a safe and economical fusion reactor. Scientific efforts to improve plasma behavior as a fusion fuel have fostered the development of a wealth of technologies and concepts for power reactors that are steadily bringing MFE to reality. Consider the unique technological needs of a magnetic fusion reactor (see Figure 2):

\section{- Confinement Technologies -} High-intensity, large-volume electromagnets to create the magnetic fields for confining the plasma, and vacuum systems that approach the low pressures found in outer space to maintain an impurity free environment for the confined plasma fuel.

- Auxiliary Plasma Heating Technologies - High-intensity, highenergy neutral beam particle injectors and radiofrequency heating systems to heat the plasma.

- Materials Technologies Materials that can withstand intense neutron bombardment, especially in the wall of the vacuum (plasma) chamber and in the energy-absorbing and fuclproducing "blanket" which surrounds the wall.

- Fueling Technologies - Continuous plasma fueling by extremely rapid injection of frozen pellets of deuterium supercooled to -269 degrees Celsius.
Conceptual reactor designs based on these evolving technologies provide a means for measuring progress against the program's technology development objectives. They also provide a framework for considering tradeoffs among technological options, economic objectives, and environmental and safety requirements.

\section{Confinement Technologies}

To construct a fusion reactor, fusion engineers must learn how best to confine an adequate amount of the reacting plasma sufficiently long that enough fusion reactions occur to produce a net power output. This involves two key confinement technologies-magnets, which create the force to confine the plasma fuel and vacuum systems, which evacuate the spent fuel and keep the fusion chamber free of impurities and spent fuel.

Large Volume Magnets - Plasma fuel, the energy source in a fusion device, is a collection of electrically charged particles which must operate at temperatures far too high for any ordinary vessel. As a solution, magnetic fields are used to confine the plasma and shape it into the forms required by the various fusion devices.

The magnetic fields required to do this are tens of thousands of times stronger than the earth's magnetic field, and they must encompass confinement chambers of large volume. Magnetic fields of this dimension are generated by complex 


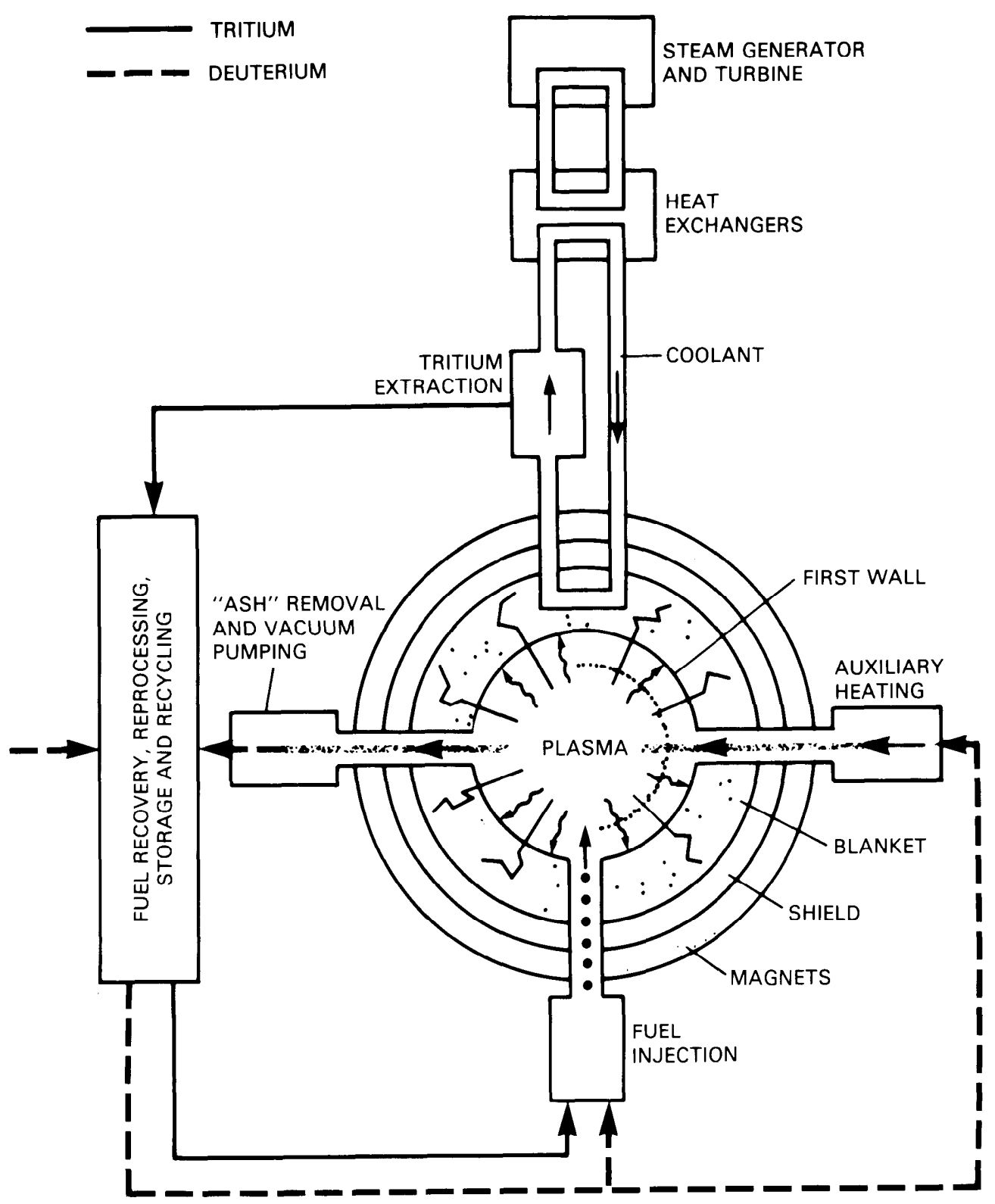

ENGINEERING PRINCIPLES common to all magnetic fusion reactors are diagrammed. A magnetic field must confine the fusion plasma; an auxiliary heating system must help to raise its temperature; a fuel-recycling system must keep it pure and well supplied with thermonuclear fuel. The heat the plasma releases must be withstood by the first wall of the reactor. The neutrons the plasma releases must penetrate into the blanket, where the energy the neutrons deposit must be transferred (in the form of heat) to a coolant. In turn the coolant can generate steam, the steam can drive turbines and the turbines can generate electricity. Nuclear reactions in the blanket must also "breed" tritium, which is radinactive and is extremely rare in nature.

From "The Engineering of Magnetic Fusion Reactors," hy Robert W. Conn. Copyright (c) 1983 by Scientific American, Inc. All rights reserved.

Figure 2 
coil systems which must be meticulously designed and constructed to withstand tremendous forces. Together with the power supplies and control subsystems, the magnet systems required for fusion rival the largest and most sophisticated electrical equipment ever constructed.

To tackle this problem, MFE scientists and engineers have developed innovative approaches to magnet design that include computer-aideddesign and other sophisticated design tools. For example, EFFI is a computerized tool created to design the complicated magnets found in tandem mirrors and other fusion devices. This computer program computes the fields, forces and inductance of magnetic coils of almost any shape. It eliminates the need to experiment or perform calculations based on scale models. It is in use not only on a National scale within the United States but is also used in Germany and Japan.

Fusion scientists have also improved magnet conductors and fabrication methods dramatically. Design tools in hand, they have found ways to use nonmagnetic materials for supporting structures, thereby leading to a major reduction of mechanical stresses within magnetic systems. One such material, Nitronic 33, is a relatively high strength nonmagnetic stainless steel alloy, which is used in the supports and cases of the TFTR's main coils. The forgings used in the TFTR are the largest ever fabricated from this material. Detailed knowledge of the mechanical and thermal environment inside a large magnet also provided the capability to establish the engineering parameters and performance goals for other innovations, such as methods to obtain high strength bonds between conductors and their insulation and new technologies for welding metals particularly adapted to use in the high-field-strength superconducting magnets.

The result of this progress is the capability to design and construct conventional and superconducting magnets of unprecedented size and power to confine the dense, high temperature plasmas into the shapes needed for fusion reactors. Magnet technology is keeping pace with program needs by building the magnets needed today and the information data base that will make it possible in the future to design, construct, and operate the magnet systems for a fusion reactor.

High Vacuum Technology - The development of techniques for large scale titanium getter pumping, the state-of-the-art in high vacuum technology for over 20 years, can be traced directly to the MFE Program.

The plasma fuel of a fusion reaction must be surrounded by a large vacuum chamber to prevent impurities from contaminating the plasma fuel and to aid in the removal of spent fuel and other fusion byproducts which could impede the fusion cycle. A full vacuum-a gaseous density $1 / 10,000$ that of air-must be maintained continuously, despite cyclic releases of spent fuel and fusion byproducts consisting primarily of hydrogen isotopes and helium. When magnetic fusion research began, conventional vacuum technology could not satisfy this requirement.

To overcome this limitation, fusion researchers had to push vacuum system technology well beyond the then state-of-the-art. Significant advances were based on the chemical concept of gettering, a mechanism by which gaseous impurities are drawn into a vacuum pump by a special chemical (a "getter") which attracts specific gaseous molecules to its surface where they are chemically bonded and thus "sucked" out of the vacuum chamber. Chemical "gettering" of gases had long been used in vacuum tubes; and although it presented a conceptual solution to the problem, no one had attempted such a large scale application of

\section{Magneform}

Magneform is a versatile process that allows metals to be welded without heat, riveted without hammering, and shaped without being touched. Hundreds of products are made or assembled using this innovative application of pulsed intense magnetic fields. These vary from metal baseball bats and sanitary caps on bottles of vaccine to automobile fuel pumps and airplane wings. Some, such as disposable cigarette lighters, would not be possible without magneformed parts.

The roots of magneform are in early magnetic fusion devices designed to "pinch" or compress the plasma to fusion conditions. Some of these devices required thin metal liners, and the magnetic fields tended to crush the liners. The fusion experimenters recognized the phenomenon's potential, patented it, and quickly redirected it to performing difficult tasks around the laboratory such as attaching connectors to large electrical conductors. Soon after, the General Atomic Company obtained an exclusive license for use of the Federally owned patent for magneform and developed a small business in magneforming.

Today, high volume assembly lines in the automotive, aerospace, energy, aircraft, electrical, defense, and other major industries throughout the world use the magneform process for fast, simple, high-quality, and economical production. Production cost savings in excess of 60 percent compared with alternative processes are achieved. The manufacture of magneform equipment is a $\$ 4$ million per year enterprise for Maxwell Laboratories, Inc. The users of magneform realize production cost savings in excess of $\$ 8$ million annually. 
getter films. Building on the getter concept, titanium getter pumps were developed (in the 1960's) to handle hydrogen loads in pulsed and nontritium machines for the next two decades. Titanium getter pumping has since been used in many of the large fusion experiments whenever clean high speed pumping for hydrogen or other chemically active gases is required.

\section{Auxiliary Plasma Heating Technologies}

Progress in plasma heating has resulted both from technological advances and greatly improved understanding of the microscopic processes underlying propagation and deposition of energy in nonuniform plasmas. Until the early 1970's, the primary technologies for heating plasmas to high temperatures were magnetic compression and resistance heating; but new technologies were needed to attain plasma temperatures for energy break-even. With new technologies such as neutral beams and radiofrequency heating developed in the 1970 's and improved in the 1980's, the heating of plasmas to break-even temperatures has become reality.

The development of neutral beam particle accelerators, high power radiofrequency systems, and microwave sources at high power and high frequency have enabled researchers to reach plasma temperatures of 200 million degrees-more than three times what had previously been possible without auxiliary heating.

Neutral Beam Particle Injectors Neutral beam heating using particle injectors is the method now used to heat plasmas to fusion temperatures. It involves the injection of high energy atoms of deuterium into the confined plasma. Before they are injected, they must be accelerated to very high energy. This is done in the following manner. Positive-charged deuterium ions or nuclei (both are the atoms stripped of its electrons) are first generated and then passed through a series of electrical grids which focuses the ions into a beam and accelerates the beam. Before the high energy ion beam passes into the fusion chamber and penetrates the confining magnetic field, the positive-charged ions are neutralized by putting a negativecharge, an electron, back on each of them.

The resulting super high energy atoms in the beam then collide with ions (nuclei) and electrons which are already present within the confined plasma. When these collisions occur, the extremely high energy of the atoms from the beam is transferred to the plasma nuclei and electrons; and their temperature rises accordingly. In the heat of the fuel chamber, the injected deuterium atoms themselves ionize into plasma, thus enriching the plasma fuel.

The research on neutral beam technology in the fusion program has precipitated qualitative advances in the understanding and manufacture of neutral beam systems. Beam performance has grown from tens of kilowatts of beam power for 10-20 thousandths of a second to tens of thousands of kilowatts of power for as long as $\mathbf{3 0}$ seconds. The development of large area sources of ions, essential to the broad uniform ion beams used by the thin film industry, materials modification industry, and semiconductor industry has been heavily influenced by the "bucket source," which was invented in the fusion program in 1975. Technology for fabricating key components-novel cathodes to cause ionization, multiaperture grids to control and focus beams, and cooling channels-have all matured significantly. Computer programs to describe beam behavior and response to focusing have also been augmented. Diagnostic instruments, such as the pinhole camera developed by the Oak Ridge National Laboratory and infrared grid scanner developed by the Princeton Plasma Physics Laboratory, lead the state-of-the-art.
Radiofrequency Plasma Heating Radiofrequency wave heating uses a steady beam of electromagnetic waves and radar waves, which are directed into the confined plasma with a special antenna. Heat energy is transferred as in a microwave oven; those particles moving at the same frequency as the beam have their temperature increased, and the temperature of the plasma goes up accordingly.

The principal engineering advantage of radiofrequency heating is the ability to locate the bulk of the equipment in an area remote from the reactor core, thus adding to reliability, simplifying maintenance, and reducing the size of the reactor hall. Other advantages include more efficient utilization of power supply, increased component life, and reduced complexity of required support equipment. In addition, radiofrequency heating offers the ability to direct the heat to specific locations in the plasma such as the center or edge, and thereby control the distribution of heat in the plasma.

The MFE Technology Development Program has led in the development of high-power gyrotrons, a class of high power microwave tubes a million times more powerful than previous ones of the same frequency. Similar technological advances have been made in components for carrying the microwave signal to the reactor hall and in high power antenna systems to send the waves into the plasma fuel. To make it possible to direct the waves from an antenna housed outside the vacuum chamber, new ceramic windows have been developed. These windows, installed in the vacuum wall, allow the waves to enter the chamber without disturbing the vacuum inside. Keeping the antenna outside the vacuum simplifies construction and maintenance of a reactor system.

Still under development, radiofrequency heating has already been used in a tokamak to attain plasma temperatures in the 50-60 million degree Celsius range. 


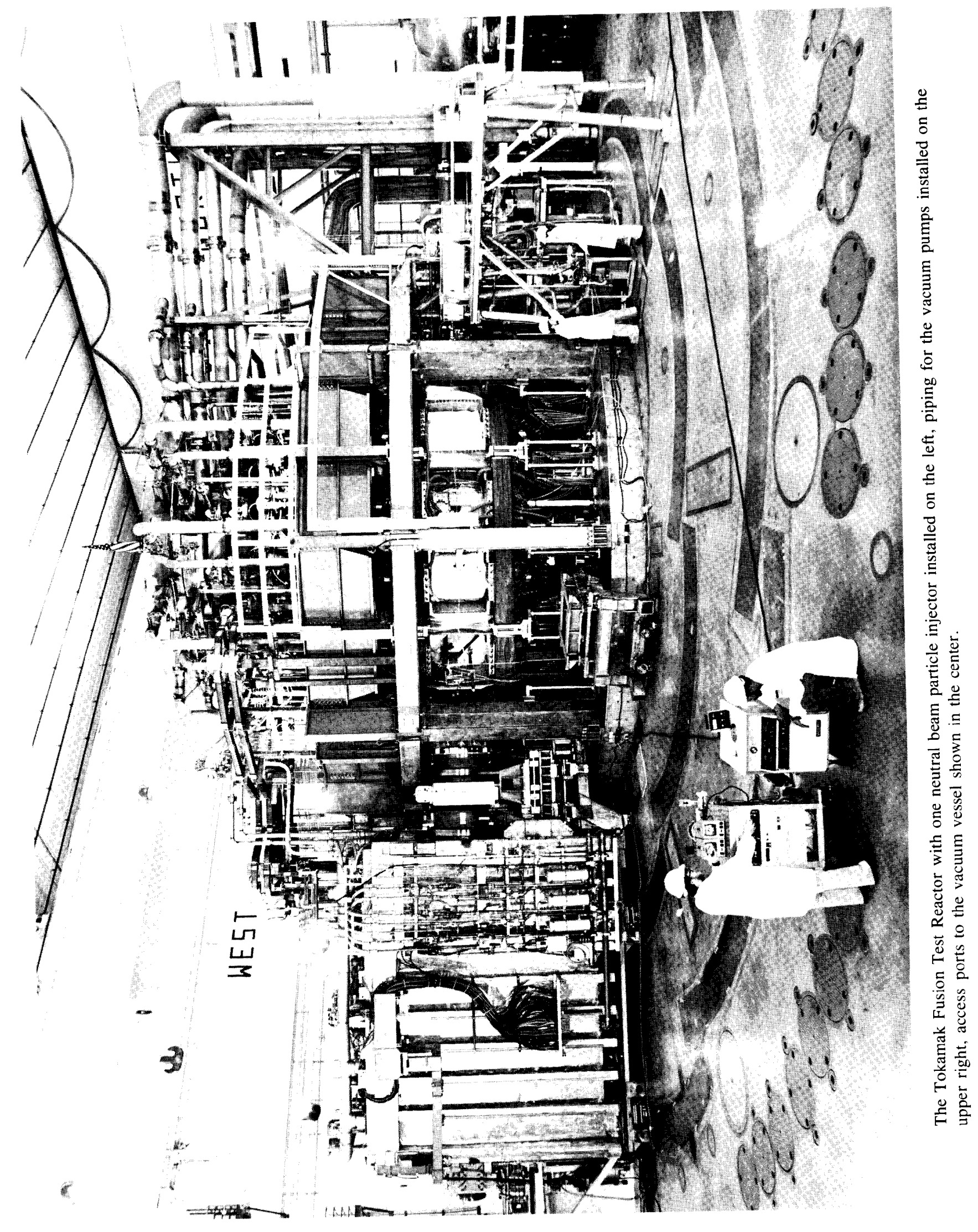




\section{Plasma Fueling Technologies}

For a sustained fusion reaction, plasma fuel must be continuously fed into the magnetic confinement chamber to replace the plasma fuel consumed by the fusion reaction. Since the confinement chamber operates within a vacuum, the original approach to plasma fueling consisted of injecting small puffs of fuel gases into the vacuum surrounding the confined plasma and allowing the materials to drift into the reaction zone. But, as the machines were scaled up, larger and denser plasmas turned out to be more difficult to fuel by gas injection from the edge because the gas could not work its way into the center of the plasma. This problem has been solved by development of a new approach based on propelling a frozen pellet of deuterium deep into the plasma. The fuel is propelled with sufficient force to penetrate the hot plasma fuel core and then melt, thereby releasing its positive ions and converting to plasma.

Building on this concept, the "pellet gun" was developed. Firing its pellets of frozen deuterium and tritium deep into the center of reactor grade plasmas, the pellet gun now provides the balanced refueling capability necessary in fusion reactors. It is a simple efficient method that is compatible with all heating methods.

Through use of the pellet gun, plasma fuels of record density have been attained, a key factor in attaining an $\mathrm{N}$-tau of 100 trillion on the Alcator $\mathrm{C}$ fusion device. So successful is this method that it is now the principal refueling technique being applied on the TFTR and the Joint European Tokamak.

\section{Materials Technologies}

Materials technology requires selecting, testing, and evaluating candidate materials for components of experimental systems and providing the scientific and engineering data base needed to do the same for commercial reactors. Materials research has led to significant advances in the identification and development of materials for service in the fusion environment. This has expanded the range of designs possible for a commercial power producing fusion

\section{Technology for National Defense}

High technology developed for magnetic fusion energy has become increasingly important for the United States National defense program. This is especially true for the so-called directed energy weapons systems. A vital segment of the technology base supporting development of neutral particle beams, electron beams, microwave beams, and free electron lasers is the product of early magnetic fusion energy research.

The technology developed for the neutral beam injectors which heat fusion plasmas to millions of degrees has been transferred to the Strategic Defense Initiative (SDI) Program where it is being modified to meet unique defense applications. The neutral beams used in the MFE program are attractive because of the extremely high beam powers which have been attained.

Both the technology and feasibility of producing powerful sharply focused beams of electrons were first demonstrated in association with an early fusion experiment called the Astron Project. It included a high current electron accelerator termed a linac, capable of injecting a tightly collimated beam of very high energy electrons into a long cylindrical vacuum vessel. The project also developed advanced techniques for manipulating such beams.

In the early 1970 's, experiments to study the propagation of these beams through gases were begun with the linac. This use of the Astron accelerator continued after the termination of the Astron experiment in 1973. Over time, the linac evolved into the Advanced Test Accelerator, the major National facility for research in electron beam weapon feasibility. The accelerator is also being used for experiments on high power microwave and laser weapons.

Microwave tubes similar to those used in plasma radiofrequency heating have been developed by the same companies for use in various military applications. Building on developments from the gyrotron program, gyroklystrons and gyro-traveling wave tubes have been produced. These oscillators and amplifiers offer advantages such as higher efficiency, compactness, high power and high frequency. For radar use, the higher frequency gives higher resolution of targets, and the higher power results in increased radar range and resistance to jamming. These characteristics are very attractive for strategic defense systems.

Another new technology is being developed using the induction linear accelerator in the Advanced Test Accelerator. The accelerator produces pulses of electrons which are passed through a magnetic "wiggler structure" to produce a laser with extremely high power levels. This socalled frec electron laser is being developed by the SDI Office and is a front runner in the current directed energy weapons research program. The free electron laser is also an important potential new heat source for plasma heating. 
reactor, and increased the potential for producing economically viable fusion power. The structural feasibility of a commercial reactor is now reasonably assured. And the scientific engineering data base needed to engineer materials for specific commercial applications and to ensure safe and environmentally acceptable sources is beginning to firm.

Increased Service Life for Structural Materials - A key component in a fusion power reactor is the blanket structure that the energy released in the fusion reactor is converted to usable heat. This conversion occurs when high energy neutrons from the fusion reactor strike the blanket. This heat is, in turn, extracted by a coolant to be converted into useful work. The structural materials that absorb the neutrons are subject to two effects: changes in mechanical properties and changes in dimensions, most often in the form of swelling. Both changes are caused by damage to the material's crystal structure by energetic neutrons. The swelling is aggravated by the agglomeration of helium (and perhaps hydrogen) produced in nuclear transmutation reactions.

When neutron damage becomes too severe, the blanket structureespecially the so called first wall that faces the plasma directly-must be replaced. The challenge when the materials research effort began in the mid-1970's was to increase predicted first wall lifetimes by at least tenfold from an unacceptable 0.4 years to 4 years or more. Predictions now are for service lives at least five times those that could be assumed in the mid-1970's, and further increases are projected. As a result, the feasibility of reactor-grade materials has been established.

Improvements in structual materials are expected because of advances over the past 10 years in understanding the fundamental processes of radiation damage and extrapolation from the growing data base on the subject. For example, it was originally predicted that the fatigue resistance of structural materials would be seriously degraded in a fusion reactor; this has been dispelled by experimentation. Substantial improvements have been made in reducing the swelling of austenitic steel, one of the primary candidate alloys for reactor structural materials. These improvements result from better alloy mixes and advanced manufacturing and processing techniques.

The question is no longer whether structural materials exist from which a commercial fusion reactor can be built; they do. For both the experimental and engineering community, the question now is how to modify known materials in ways to decrease cost, improve performance further, and minimize waste disposal requirements.

Of particular interest are structural materials that minimize residual induced radioactivity so that they can be disposed of safely without the long-term hazard associated with fission waste. Here, too, much progress has been made toward practical choices of first wall and structural materials that can be disposed of by shallow burial, perhaps at the plant site. Research on such low waste alloys will continue to be an important component of fusion materials research.

Durable Magnet Materials - At the beginning of the fusion program, information was needed about the maximum tolerable neutron exposure for superconducting magnet components: the superconductor, the stabilizer, and the insulator. Without this information, early reactor designs had to assume poor radiation tolerance and accommodate massive and costly shielding to protect the magnets. Based on current tests, the fusion materials program has now confirmed that the superconducting materials with the best operating characteristics for fusion magnets (NiobiumTitanium and Niobium-Tin) are sufficiently durable for magnets to function as intended with much less shielding than was thought possible earlier in the program. This will lead to savings of millions of dollars in construction costs for a power reactor.

High Performance First Wall Materials - Even though the plasma fuel is confined in a "magnetic bottle" during normal operation, it still drifts outward until it touches and is neutralized on "limiters," protecting the vacuum chamber walls. There the particles dislodge atoms or other particles, which enter the plasma as impurities. Once in the plasma, the impurities act like wet wood thrown on a fire. They absorb and radiate energy intended to heat the plasma without contributing to the energy producing process. To make matters worse, impurity atoms (which do not react) dilute the fuel plasma. The heavier the impurity atom, the worse the energy drain.

By 1978 energy losses due to impurities became the critical impediment to raising plasma to the temperatures required for energy breakeven. A solution came from the understanding of the plasma-edge environment that had been accumulating in plasma physics studies, and from the knowledge gathered in materials research about the interaction between the plasma particles and materials. Knowledge of the mechanical and thermal stresses on the limiters led to a search for superior materials to limit the plasma. The answer was to fabricate the limiters out of carbon in place of the heavier refractory materials which were in use at the time.

The change in material for limiters was responsible for a 30 million degree Celsius increase in plasma temperature on the PLT.

Ceramics for Insulation and Fuel Breeding - Ceramics are required for a variety of insulator applications in a fusion reactor, some of which will receive high neutron exposure. In 
addition, ceramics containing lithium are promising candidates for tritium breeding materials.

When the fusion materials program began, the little information available on radiation effects in ceramic insulators suggested that poor performance could be expected. This meant that reactor designs would have to accommodate the need for frequent replacement of key components. The fusion ceramics testing program has since demonstrated that a number of ceramics offer extended lifetimes in fusion reactor applications. Furthermore, increased understanding of degradation mechanisms has led to effective methods of enhancing radiation resistance.

The importance of ceramics takes on added dimensions in light of promising indications of their usefulness in breeding tritium in the blanket. If the blanket contains lithium, some of the neutrons striking it will interact with the lithium to produce tritium, a constituent of reactor fuel that is not found in nature. Since lithium is difficult to handle in pure forms, a solid lithium compound such as a ceramic containing lithium could offer considerable advantages. Previous doubts that tritium could be successfully extracted from a solid compound have been dispelled following an experiment in which tritium was produced from a lithium ceramic compound while it was being bombarded with neutrons from a fission reactor. It has also been shown that ceramic compounds will hold up adequately under the neutron bombardment of the fusion environment.

\section{Reactor Concepts}

For the first time in the history of fusion research, there seems now to be a substantial and reliable experimental basis for the detailed description of the fundamental scientific and technology requirements of a magnetic fusion reactor. The key issues have gradually been revealed by the detailed series of conceptual reactors designed and produced within the MFE Program over the last 15 years. The object of these studies is to describe a plausible fusion reactor based on the underlying physics and reasonable extrapolations of the technology.

Research and design studies on a broad front have made it possible to describe the forms that commercial fusion reactors might take. The re- quired technologies, the plant power balance, the capital and operating costs, and the safety and environmental features of fusion power can all be estimated. A reactor study is performed by a multidisciplinary team of physicists and engineers that must keep one eye on present-day experiments and theory and one eye to the possible future reactor end-product. The feedback from this study then steers it towards the safest and the most environmentally benign application of fusion energy.

These studies allow an early assessment of the potential and impact of fusion reactors in the economy. They project that capital costs could be acceptable, that electricity could be produced at around 50 mills/ $\mathrm{Kwh}$, and that the environmental and safety features of fusion could make it more attractive than other long range energy options.

For the past 15 years, these studies and assessments have helped guide the present scientific and technology development programs, determine the directions for an improved fusion product, and provide a technical assessment of the fusion energy option for use in long range energy planning. 
The United States is participating in international programs for the development of magnetic fusion. Incentives for international cooperation include the enhancement of progress through the pooling of multidisciplinary expertise and achievement of significant cost reductions in technology development through cost-sharing. In pursuing collaboration, the United States is proceeding so as to enhance National Security in the full sense of the term including energy, economic and defense security. This requires maintenance of sovereignty over our own National fusion program. All international programs are frequently reassessed to assure their continuing value in this sense.

An instance of international cooperation having an especially crucial impact on the MFE Program was the dissemination by the Soviets of the tokamak confinement concept in the 1960's. Knowledge of this innovation altered the entire course of United States fusion research and has led to inestimable savings of program resources.

The fusion development community consists of four primary blocs: the United States, the USSR, Japan, and the European community. There are also two important international agencies that provide an avenue for scientific exchange: the International Energy Agency (IEA) and the International Atomic Energy Agency (IAEA).

Cooperative activities began with what is now the longest standing, most traditional element-personnel exchanges, in which individuals contribute their knowledge and experience in joint discussions with colleagues in other countries. The international dimensions of the MFE Program have since expanded from academically oriented information exchanges to encompass coupled experiments, jointly funded scientific and technology research facilities and plans for multilateral cooperation in the building of the next generation tokamak reactor.

Research enhancements worth more than $\$ 100$ million to the United States have resulted from international cooperative research in the areas of materials research, superconducting magnet development, plasma wall interactions, upgrade and operation of the Doublet III (DIII) concept definition, and design of the next generation tokamak experiment (International Tokamak Reactor (INTOR)).

\section{Superconducting Magnet Development}

The United States, the European Atomic Energy Community, the Japan Atomic Energy Research Institute, and the Swiss Institute for Nuclear Research cooperate under the auspices of the IEA on superconducting magnet development in the Large Coil Task. The International Fusion Superconducting Magnet Test Facility, which the United States planned and constructed in Tennessee in consultation with the other participants at a cost of $\$ 40$ million, is used in the development of the next generation of superconducting magnets. Each 
of the participants designed and constructed one or more of the six test coils to be tested in the facility. Results from the Large Coil Task will be used in the coil design for the next large tokamak device.

\section{Plasma/Wall Interactions}

Plasma interactions with the confinement chamber wall create impurities which interfere with the fusion reaction. Advances in overcoming this problem have been made previously through informal international collaboration which led to a formal international cooperative research program on minimizing the effect of plasma-wall interactions. Participants include the United States, the European Community, Japan, and Canada, under the auspices of the IEA. The United States is completing the design of an innovative pump limiter for improved impurity control that will be built by the Japanese according to United States specifications. It will then be tested on the TEXTOR tokamak in West Germany. The TEXTOR tokamak was selected over other sites because it has been designed for studies of plasma/wall interactions.

\section{Doublet-III}

In 1979 the United States and Japan agreed on a multi-year Doublet III project seeking to investigate the possibility of improving the perform- ance of plasma fuels in tokamaks with noncircular cross sections. The Japan Atomic Energy Research Institute was to provide funds for upgrading the Doublet III facility for operation with increased magnetic field, plasma current, and neutral beam power. By 1984 Japan had invested $\$ 60$ million in the Doublet III program and trained the operations team for their subsequent experiment on Japan's JT60 fusion device.

The original bilateral agreement has since been amended to include a modification of the vacuum vessel to a D-shape to achieve greater plasma performance capability. The agreement has been extended to 1988 , with the United States and Japan contributing $\$ 10$ million and $\$ 8.6$ million, respectively.

\section{International Tokamak Reactor}

Some form of international collaboration on large facilities may become a necessity in the future since a fusion test reactor is currently estimated to cost more than one billion dollars.

The INTOR Workshop was initiated collaboratively by the United States, USSR, Japan and the European Community under IAEA auspices in 1979 to proceed jointly in phases from feasibility assessment through concept definition and design to construction and operation of the next generation major tokamak experiment.
The first two planned phases of feasibility assessment and concept definition were completed in 1979 and 1981. Subsequent phases of the INTOR activity have emphasized the focusing of worldwide effort on critical technical issues affecting such a large undertaking, rather than on detailed design and construction. As a result, the INTOR activity has substantially advanced international understanding of a number of critical technical issues and stimulated interest in investigating the feasibility of potential improvements.

\section{Materials Research}

The Rotating Target Neutron Source-II, built by the MFE Program for fusion research, is the world's most intense fusion neutron source dedicated to fusion materials research. Scheduled for shutdown in 1987, it has for about 5 years been the center of a cooperative United States and Japanese project that is now validating theories predicting how fusion neutrons will affect materials in reactor components.

Each country is contributing about $\$ 2$ million annually for full operation and experimental utilization of the Rotating Target Neutron Source II facility in the United States. Japan and the United States also are spending about $\$ 1$ million annually to conduct neutron irradiation tests in the High Flux Isotope Reactor and the Oak Ridge Reactor. 